\title{
Application of Shape From Shading Technique for Side Scan Sonar Images
}

\author{
Krzysztof Bikonis, Ph. D. \\ Marek Moszynski, Assoc. Prof. \\ Zbigniew Lubniewski, Ph. D. \\ Gdansk University of Technology, Poland
}

\begin{abstract}
Digital signal processing technology has revolutionized a way of processing, visualisation and interpretation of data acquired by underwater systems. Through many years side scan sonars were one of the most widely used imaging systems in the underwater environment. Although they are relatively cheap and easy to deploy, more powerful sensors like multibeam echo sounders and sonars are widely used today and deliver $3^{D}$ bathymetry of sea bottom terrain. Side scan sonar outputs data usually in a form of grey level $2^{D}$ acoustic images but the analysis of such pictures performed by human eye allows creating semi-spatial impressions of seafloor relief and morphology. Hence the idea of post-processing the side scan sonar data in a manner similar to human eye to obtain $3^{D}$ visualisation.

In recently developing computer vision systems the shape from shading approach is well recognized technique. Applying it to side scan sonar data is challenging idea used by several authors. In the paper, some further extensions are presented. They rely on processing the backscattering information of each footprint (pixel in sonar image) along with its surroundings. Additionally, a current altitude is estimated from the size of shadow areas. Both techniques allow constructing $3^{D}$ representation of sea bottom relief or other investigated underwater objects.
\end{abstract}

Key words: side scan sonar data processing; shape from shading

\section{INTRODUCTION}

Acoustic sensors offer robust insight into underwater environments, as they are characterised by longer range and wider angle coverage compared to video cameras or other sensors. Also, they are capable of providing satisfactory results of mapping the environment in turbid waters. Side scan sonar (SSS) is one of the most widely used imaging systems in underwater environment. It is relatively cheap and easy to deploy, in comparison to more powerful sensors like multibeam sonars. However, it has some limitations, such as its inability to recover the seafloor depth or submerged object information directly [1].

Conventional side scan sonars are used until today. They emit fan-shaped pulses down toward the seafloor across a wide angle perpendicular to the path of the sensor through the water, which may be towed from a surface vessel or mounted on the ship's hull. The intensity of the acoustic reflections from the seafloor of a fan-shaped beam is recorded in a series of crosstrack slices. When stitched together along the direction of motion, these slices form an image of the sea bottom within the swath of the beam.

During years of evolution side scan sonar were improved including higher frequency systems and the dual-frequency sonars, and the combined side-scan and sub-bottom profiling sonar. Generally, increasing frequency improves resolution of obtained images. The development of digital signal processing technology made possible real time processing of acquired data to form synthetic aperture. The principle of such synthetic aperture sonars (SAS) is to move a sonar along a line and illuminate the same spot on the seafloor with several pings. This produces a synthetic array equal to the distance traveled. By coherent reorganization of the data from all the pings, a synthetic aperture image is produced with improved alongtrack resolution. In contrast to conventional side scan sonar, SAS processing provides range-independent along-track resolution. At maximum range the resolution can be magnitudes better than that of side scan sonars.

Until the development of so-called 3D side scan sonars the side scan sonar imagery were presented in the form of classical $2^{\mathrm{D}}$ images. $3^{\mathrm{D}}$ side scan sonars uses interferometric acoustic technology and by application of digital signal processing techniques it operates over a field of view larger than $90^{\circ}$ with no blind spots under the survey track.

However, many SSS 2D images exist, that could be transformed into $3^{\mathrm{D}}$ representation in an algorithmic way using the echo intensity information contained in grayscale images. In the imaging sonar systems, the characteristics of acoustic 

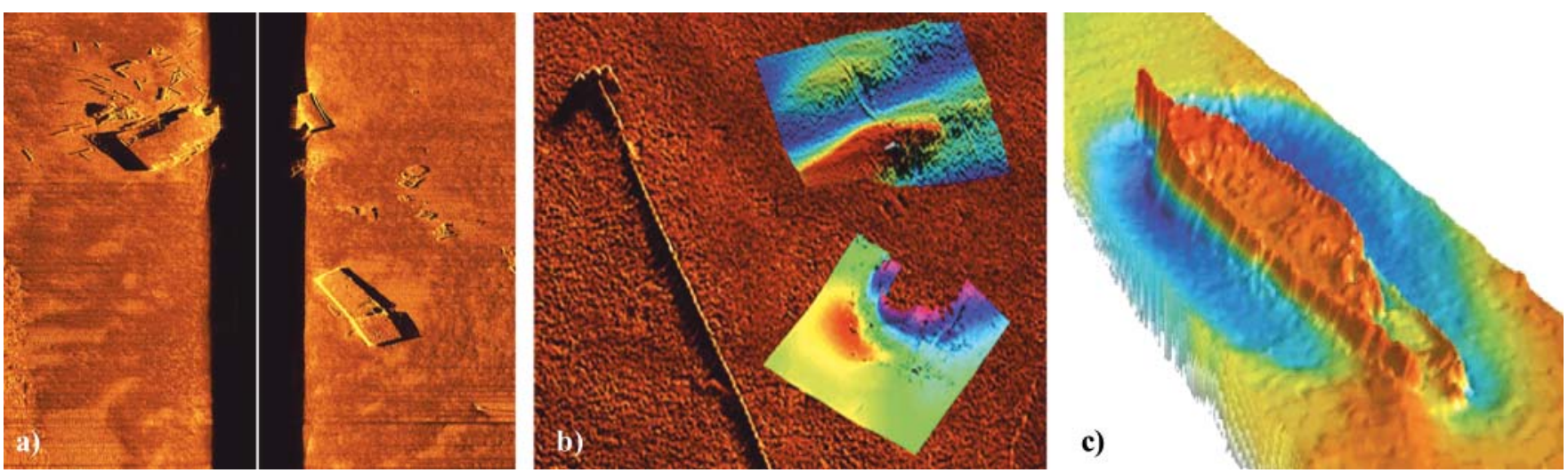

Fig. 1. Sample sea bottom images obtained by three generations of side scan acquisition systems a) conventional side scan sonar b) synthetic aperture sonar c) $3^{D}$ side scan sonar

energy backscattering by a target localised on seafloor is utilised. Instead of arbitrarily using the formula like Lambert's law to describe the seabed backscattering dependence on an incident angle [2], the authors propose their own approach to derive this dependence locally from experimental data.

In this paper, two methods for 3D seafloor and submerged objects shape reconstruction from SSS images, based on shape from shading (SFS) approach, are presented. In the first method, for estimation of bottom local depth at a given pixel of sonar image, the information from both currently processed and previous ping is utilised. It allows the local seabed surface element orientation to have two degrees of freedom. Prior to the investigation of this approach on sidescan sonar field data, its verification on multibeam sonar backscatter data is presented, with use of the seabed $3^{\mathrm{D}}$ model (i.e. the set of (x, $\mathrm{y}, \mathrm{z}$ ) seabed surface points derived from multibeam soundings) as a reference.

The second method is used for $3^{\mathrm{D}}$ submerged object shape reconstruction and its imaging. The direct application of classical SFS technique seems not to be best suited to submerged object visualisation as it leads to obtaining very smooth shapes which differ significantly from actual forms of artificial objects. The proposed method, besides the utilisation of the local altitude gradient estimation by SFS algorithm with use of the backscattering coefficient angular dependence function derived experimentally, also estimates the elevation change on the basis of the dimension of acoustic shadow areas.

Several techniques of 3D geometry reconstruction for seabed surface or submerged objects using side-scan sonar images has been reported $[3,4,5]$. Mainly, they use the techniques based on the problem inverse to image formation, namely Shape from Shading (SFS), which is one of classical problems in computer vision (see [6] for a collection of significant papers on SFS). The goal is to derive a $3^{\mathrm{D}}$ scene description from one or more $2^{\mathrm{D}}$ images. Given a grey level image, the aim is to recover the light source and the surface shape at each pixel of the image.

Brief description of the SFS principles and basic methods along with literature references is given in [7].

\section{SHAPE FROM SHADING FOR SIDE SCAN SONAR IMAGERY}

\section{Seafloor relief reconstruction from sonar image}

In the construction of a seabed elevation map from side scan images, the SFS technique relays on calculating the local slope of bottom relief, given the image pixel intensity, the assumed dependence of bottom surface backscattering coefficient on incident angle (what corresponds to reflectance map in classical SFS), and the estimated local incident angle value. The presented algorithm for $3^{\mathrm{D}}$ seafloor relief reconstruction [8] has been developed assuming that the acoustic reflectivity model as well as the altitude $\mathrm{H}$ of the sonar transducer are given. The intensity (grey level) of a pixel in sonar image is treated as proportional to the acoustical intensity of backscattered echo. Also, the simple, straight line propagation path of acoustic wave in water column is assumed, and, the dimensions along vertical axis $(\mathrm{z})$ of an object to be reconstructed are assumend to be small in comparison with the sonar transducer altitude.

The geometry used in derivation of the reconstruction algorithm is presented in Fig. 2. The beam of a side scan sonar covers an angular sector from $\varphi_{\min }$ to $\varphi_{\max }$.

The relation between the time instant $t_{i j}$ in an echo envelope and the across-track coordinate $\mathrm{x}_{\mathrm{j}}$ of a corresponding point $\mathrm{P}_{\mathrm{ij}}$ on seabed surface, may be expressed by:

$$
x_{j}=\sqrt{\left(\frac{c_{i j}}{2}\right)^{2}-H^{2}}
$$

where:

$t_{\mathrm{ij}} \geq 2 \mathrm{H} / \mathrm{c}$
$\mathrm{c}-$ sound speed in water.

At the time instant $t_{i j}$, the seafloor surface $S_{i j}$ is insonified, the area of which may be for a flat bottom case expressed by the classical equation [2]:

$$
S_{i j}=\theta_{V} R_{i j} \frac{c \tau}{2 \sin \theta_{i j}}
$$

where:

$\Theta_{\mathrm{V}}-$ the along tract transducer beamwidth,

$\mathrm{R}_{\mathrm{ij}}$ - the range from the transducer to the point $\mathrm{P}_{\mathrm{ij}}$

$\tau$ - the transmitted pulse length.

The 3D bottom relief was reconstructed by estimation of an altitude $\mathrm{z}(\mathrm{x}, \mathrm{y})$ sequentially for consecutive discrete points $(\mathrm{x}, \mathrm{y})$ on a plane, using the scheme depicted in Fig. 2. For the $(i, j)$ iteration (where $i$ - number of processed line in the sonar image corresponding to one sonar ping, $j$ - number of pixel belonging to this line $)$, i.e. the point $\mathrm{P}_{\mathrm{ij}}=\left(\mathrm{x}_{\mathrm{j}}, \mathrm{y}_{\mathrm{i}}, \mathrm{z}\left(\mathrm{x}_{\mathrm{j}}, \mathrm{y}_{\mathrm{i}}\right)\right)$ altitude estimation, the local triangle facet was being taken into account, with vertices at two previously estimated points $\mathrm{P}_{\mathrm{i}-1 \mathrm{j}}=\left(\mathrm{x}_{\mathrm{j}}, \mathrm{y}_{\mathrm{i}-1}, \mathrm{z}\left(\mathrm{x}_{\mathrm{j}}, \mathrm{y}_{\mathrm{i}-1}\right)\right)$ and $\mathrm{P}_{\mathrm{i}-1}=\left(\mathrm{x}_{\mathrm{j}-1}, \mathrm{y}_{\mathrm{i}}, \mathrm{z}\left(\mathrm{x}_{\mathrm{j}-1}, \mathrm{y}_{\mathrm{i}}\right)\right)$, and currently estimated point $\mathrm{P}_{\mathrm{ij}}$. Using the applied model, the value chosen for $\mathrm{Z}_{\mathrm{ij}}$ allows for calculation of normal $\overrightarrow{\mathrm{N}}_{\mathrm{ij}}$ to the surface facet, the angle $\Theta_{\mathrm{ij}}$ and the local intensity $\mathrm{I}_{\mathrm{ij}}$ value, which then many be compared with that from the original sonar image. 


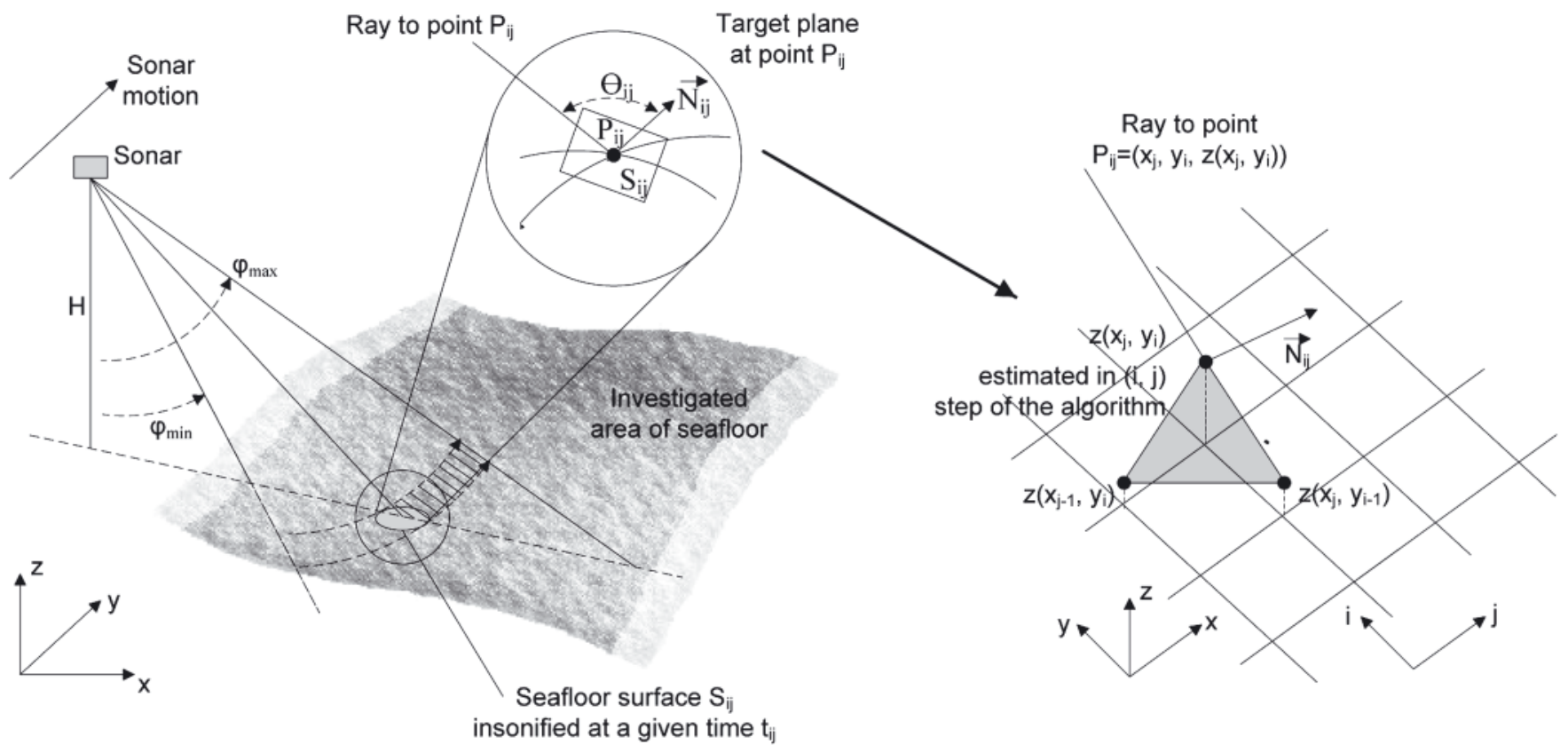

Fig. 2. The geometry used in derivation of the seafloor relief reconstruction algorithm and its grid approximation

Optimal $z_{\mathrm{ij}}$, i.e. that giving I equal to a measured value, may then be estimated [7].

The reliable reflectance model (namely, here: the seafloor acoustic backscattering coefficient dependence on an incident angle) is a crucial element of the SFS-like 3D object reconstruction procedure. Some authors suggest that the Lambert's Law provides a good fit to seabed backscattering dependence on an incident angle [2], but in general, the description of seafloor backscattering phenomenon is complicated. The backscatter from the seafloor is generally considered to be composed of a combination of surface and volume scattering, i.e. roughness interface scattering and scattering from inhomogeneities within the sediment volume. The detailed investigation of seafloor backscattering angular dependence, containing the theoretical modelling along with experimental verification, is described in [9]. It shows that although in several cases the application of Lambert Law might be allowed, in many others this law is not satisfied. This fact was also proved by further experiments. Taking it into account, the authors proposed their own approach here, which assumes the estimation of backscattering angular dependence coefficient locally for currently used experimental data. Assuming that we may choose the region within the processed sonar image, where the seabed surface is flat and composed of the material characterised by constant properties, we may state that the grey level of a given pixel in an analysed area should depend only on the experiment geometry (e.g. position of the source, the incident angle), the sonar calibration data, and the seafloor backscattering coefficient. If the investigated area covers some range of incident angle values, the backscattering coefficient angular dependence may be estimated for this range using pixel grey level values, as it is presented in the next section.

\section{The use of shadow area dimension for object height estimation}

One extension has been made for the proposed $3^{\mathrm{D}}$ shape reconstruction algorithm from SSS data when used to artificial objects lying on seabed (like wrecks for instance). Such object usually have regular shapes and their sonar images often are full of shadow areas. The proposed algorithm extension assumes the utilisation of the information which may be derived from shadow areas. Namely, the height of an object element is being estimated from the size of a current shadow area. Shadow area include the pixels from SSS image witch values are below chosen threshold intensity value $\mathrm{I}_{\mathrm{th}}$. In a case of a shadow zone detection of a length $\mathrm{j}$ along $\mathrm{x}$ axis, the altitude values from $z_{i+1}$ to $z_{i+j}$ were set to unknown, and the $z_{i+j+1}$ value was calculated as:

$$
z_{i+j+1}=z_{i}-\frac{\Delta x_{s h}}{\tan \varphi_{i}}
$$

Fig. 3 presents in a schematic way the influence of the $\mathrm{I}_{\text {th }}$ on the algorithm results for two cases of $\mathrm{I}_{\mathrm{th}}$ values. Figure $3 \mathrm{a}$ shows the sample dependence of the intensity value on $\mathrm{x}$ coordinate for a given fragment of one line in side scan sonar image, along with two $\mathrm{I}_{\text {th }}$ values indicated. Figure $3 \mathrm{~b}$ presents the reconstructed seabed altitude for these two cases. For $I_{\text {th2 }}$ case, the larger shadow zone occurs and no reconstruction is obtained inside $\left[\mathrm{x}_{\mathrm{a}}, \mathrm{x}_{\mathrm{b}}\right]$ range.

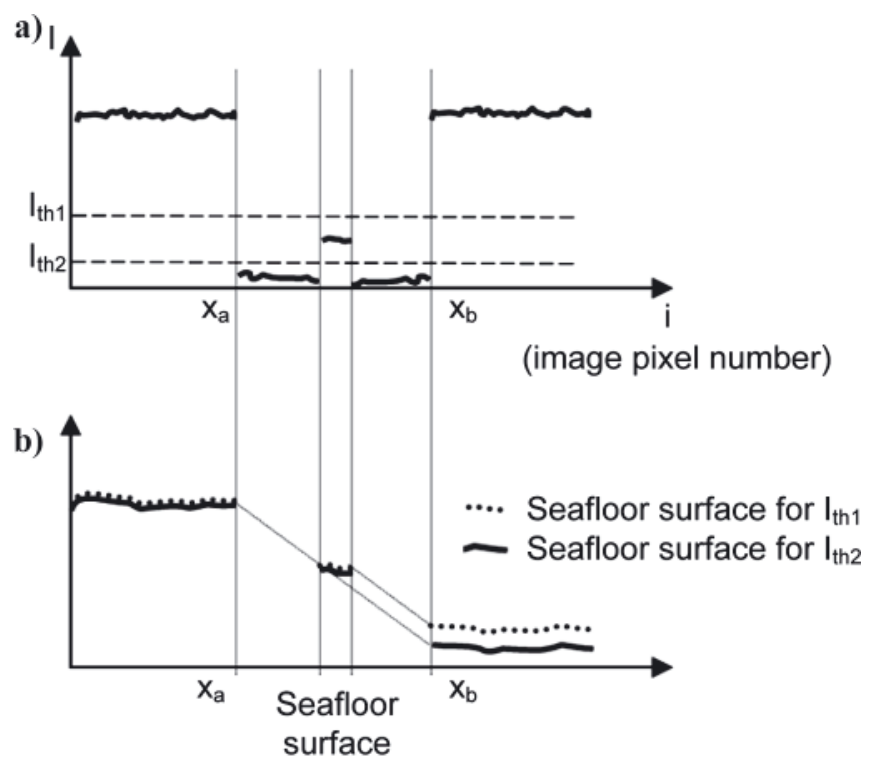

Fig. 3. The influence of the shadow threshold value $I_{\text {th }}$ on the altitude reconstruction algorithm results 

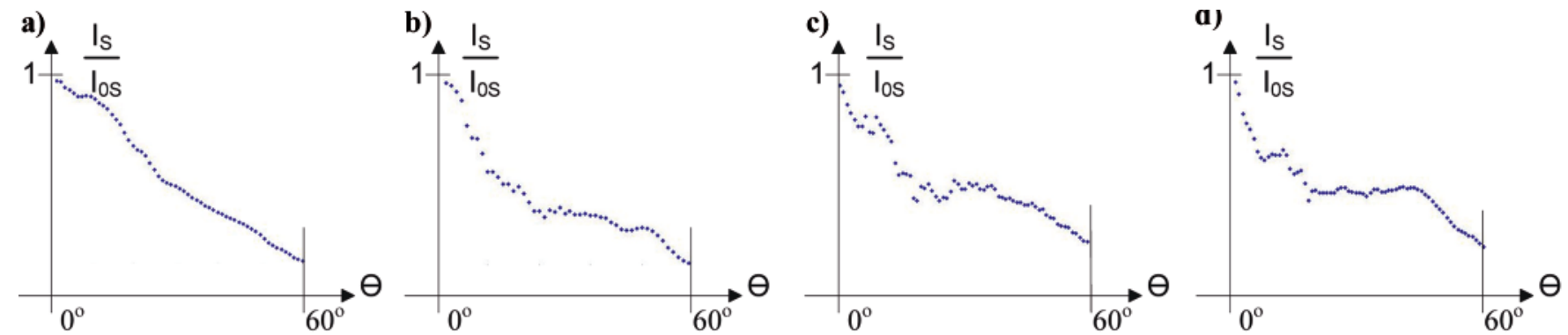

Fig. 4. Seabed backscattering coefficient angular dependence averaged for 40 multibeam soundings for each of 4 sites

a)
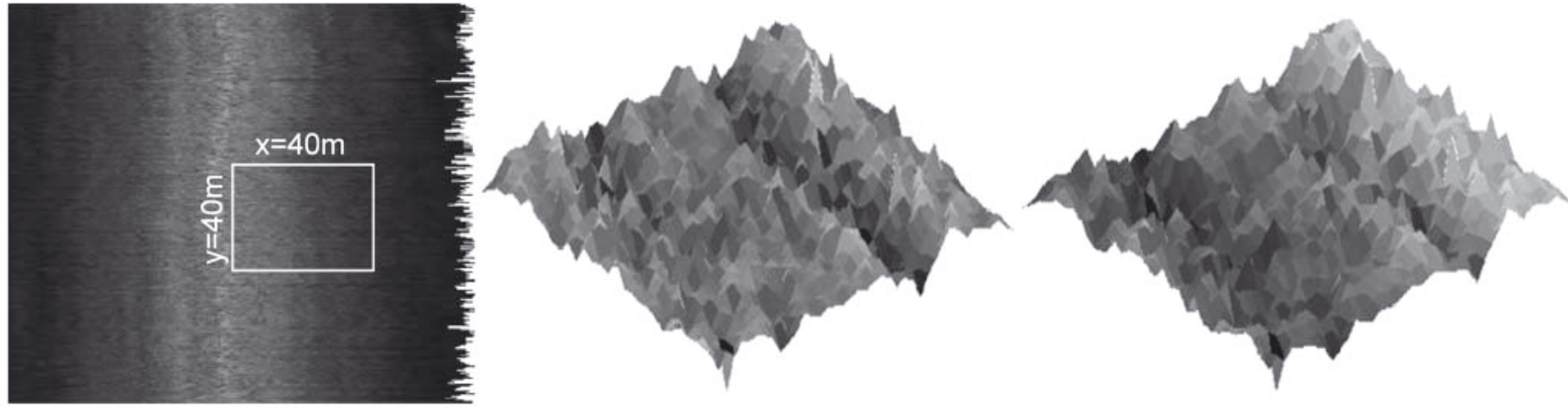

b)
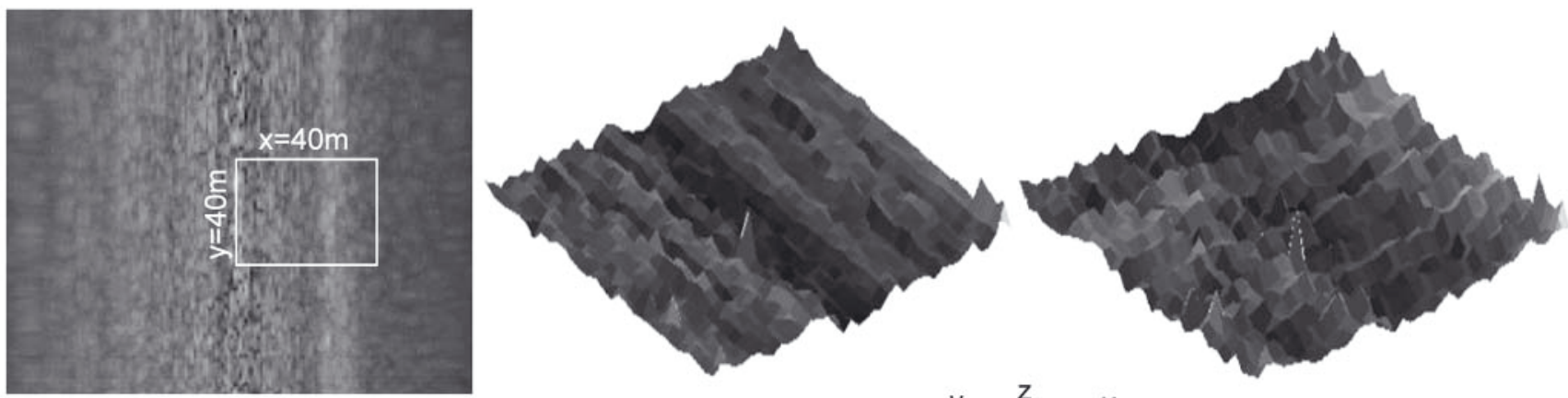

c)
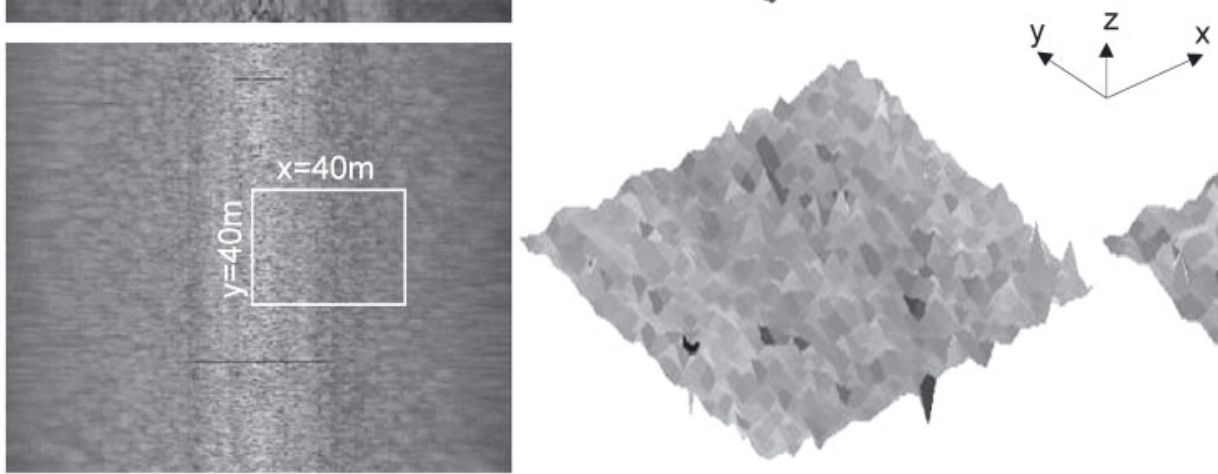

d)
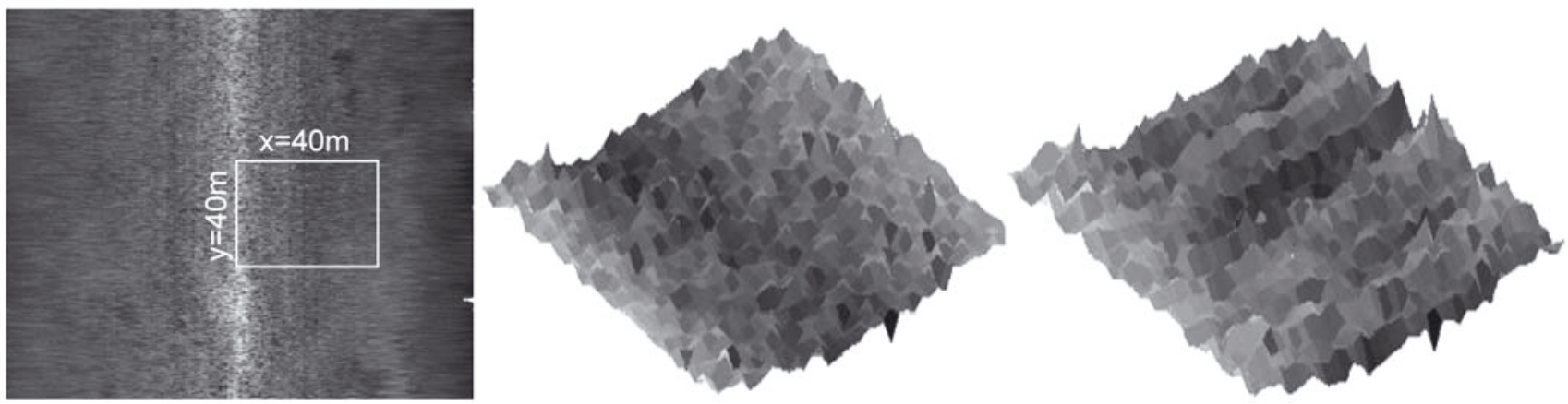

Fig. 5. Verification results of $3^{D}$ shape reconstruction method using multibeam sonar data for 4 sites in Gdansk Bay region. First column: sonar images constructed using backscattering strength of echoes corresponding to several multibeam soundings (horizontal lines in an image) and beams (vertical lines in an image). Central column: visualisations of seabed $3^{D}$ models derived from multibeam soundings by the EM3002 sonar software in the form of sets of $(x, y, z)$ seabed surface points - these seabed surfaces

were used as a reference data. Right column: $3^{D}$ seabed surface shapes reconstructed using the proposed SFS-like algorithm 


\section{EXPERIMENTS AND RESULTS}

\section{Experimental derivation of backscattering coefficient angular dependence}

As it has been introduced in the previous section, the seafloor backscattering coefficient angular dependence function, needed in the SFS-like 3D shape reconstruction procedure, has been derived experimentally by choosing flat surface area for this purpose.

The data used in this experiment were acquired by the Kongsberg EM 3002 multibeam sonar in Gdansk Bay region of the Baltic Sea for 4 separate sites characterised by different seafloor type. The sonar operating frequency was $300 \mathrm{kHz}$, the width of beams: $1.5^{\circ} \times 1.5^{\circ}$, the transmitted pulse length: 0.15 $\mathrm{ms}$, the echo sampling rate: $14.3 \mathrm{kHz}$. The bottom depth was in a range approximately between $10 \mathrm{~m}$ and $100 \mathrm{~m} .500$ swaths from each of four seafloor types were processed. For each swath, 160 beams covered the angle sector from $-65^{\circ}$ to $65^{\circ}$.

Fig. 4 presents the seafloor backscattering coefficient angular dependence derived by the described approach and averaged for 40 multibeam soundings for each of 4 sites. The significant differences in the results between several cases are well visible. It proves the expectation that this dependence characterises the seabed locally and therefore it should be preferable to derive it experimentally in-situ.

\section{Verification of the shape reconstruction procedure using multibeam sonar data}

The mutibeam data described in the precious subsection were also used in the verification procedure for the proposed algorithm of $3^{\mathrm{D}}$ shape reconstruction from acoustic data. The obtained results are presented in Fig. 5 for 4 different acquisition sites in Gdansk Bay. The seabed "sidescan sonarlike" images constructed from multibeam backscatter data (first column of pictures in Fig. 5) were processed by the proposed SFS-like algorithm to reconstruct the seafloor relief. The results are shown in the right column of pictures in Fig. 5 while the reference data, i.e. 3D seabed surfaces derived from multibeam data by EM3002 sonar software, are shown in the central column in this figure.

It is actually visible in Fig. 5 that although the proposed method introduces some "artifacts" in across-track direction (from bottom left to top-right on the pictures in Fig. 5), the differences between the reconstructed and the reference seabed surfaces are small enough to be primarily acceptable. For the quantitative estimation of the proposed method performance, the rms difference between the reconstructed and the reference surface has been calculated for each of 4 cases. The obtained rms values were between $3 \mathrm{~cm}$ for the first site (a) and $9 \mathrm{~cm}$ for the last site (d) what is a good result taking into account that the geometric dimensions of the experiment were of tens of meters.

\section{Application of the $3^{D}$ shape reconstruction procedure to side scan sonar data}

The developed procedure of $3^{\mathrm{D}}$ seafloor relief reconstruction was also tested on DF1000 dual frequency (100 and $500 \mathrm{kHz}$ ) side scan sonar data collected in the Gulf of Gdansk. For further processing the $500 \mathrm{kHz}$ data were used as they represent the higher resolution entity. The specific areas were selected for calculation of seabed backscattering coefficient angular dependence. The results of $3^{\mathrm{D}}$ seabed surface reconstruction are presented in Fig. 6.

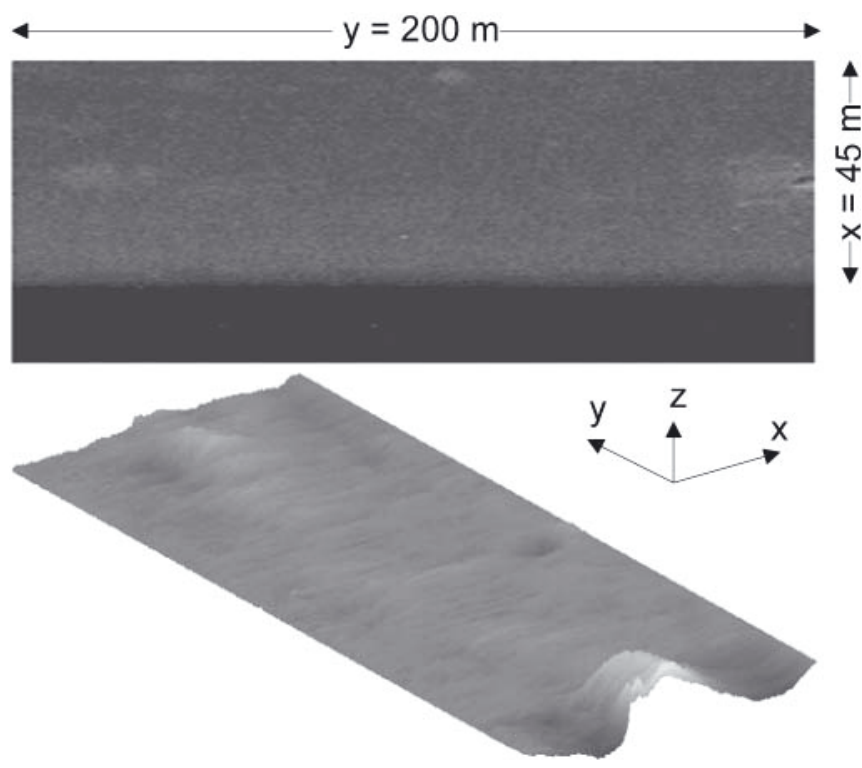

Fig. 6. The sample seabed image with selected areas used for seabed backscattering calculation [10]

The developed procedure of object $3^{\mathrm{D}}$ shape reconstruction was tested on side scan sonar data downloadable from Marine Sonic Technology, Ltd. website. The sample car tire image acquired by side scan sonar is presented in Fig. 7 and its reconstruction is shown in Fig. 8.

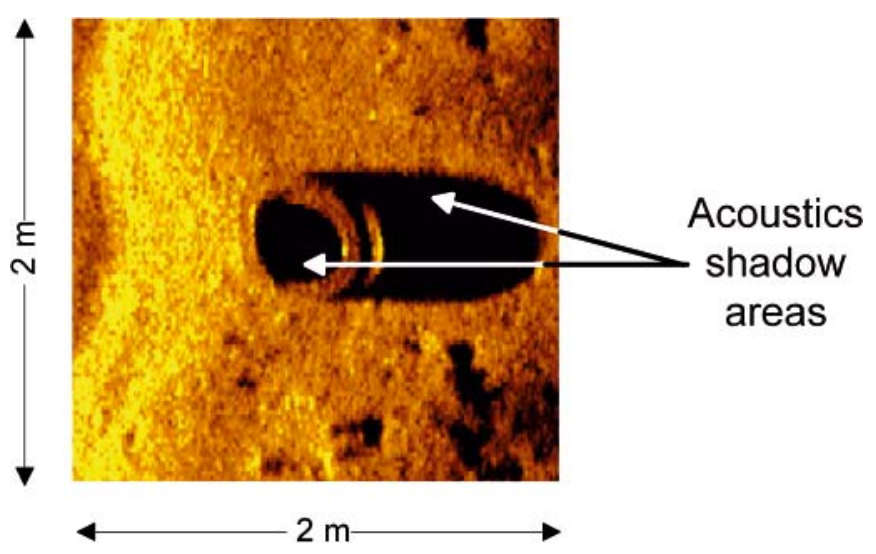

Fig. 7. Side scan sonar image of car tire from sonar operated at $2400 \mathrm{kHz}[10]$

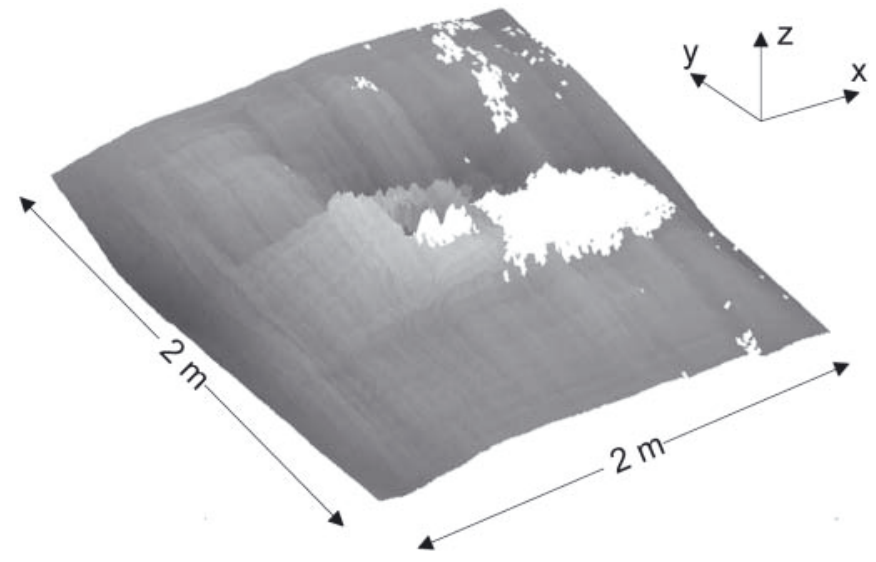

Fig. 8 . The $3^{D}$ car tire reconstruction

The next test was performed on side scan sonar data containing the image of USS Utah as shown in Fig. 9. The image region within the rectangle was used to verify the performance of the algorithm. 


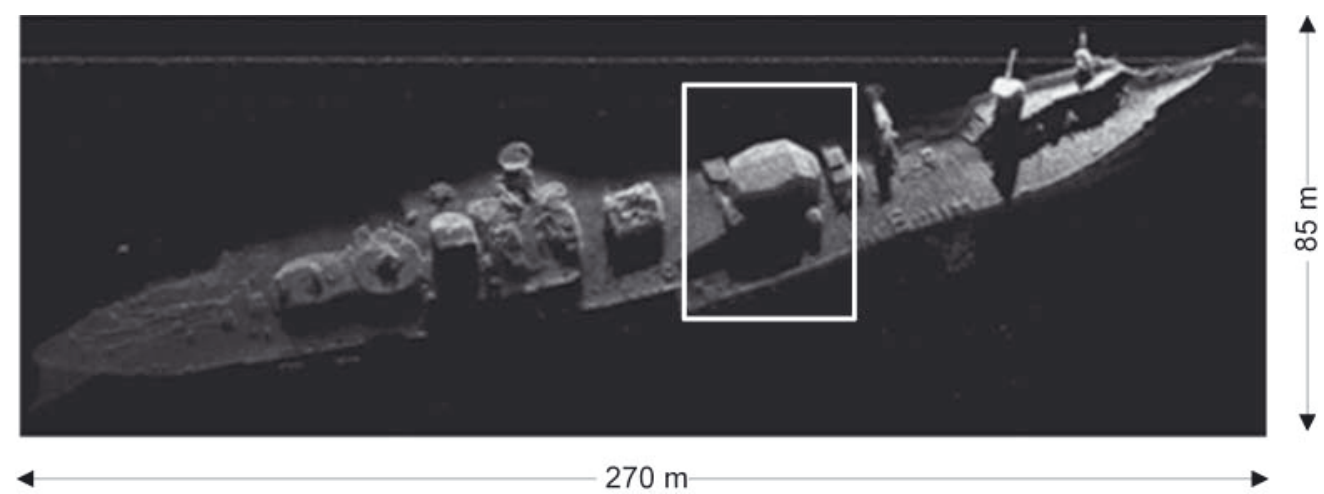

Fig. 9. Image of the USS Utah, resting in Pearl Harbor near Ford Island, acquired by the U.S. Army $7^{\text {th }}$ Engineer Detachment using Sea Scan Centurion system operated at $600 \mathrm{kHz}$ [10]

The comparison of the results obtained when applying two different shadow threshold values $\mathrm{I}_{\mathrm{th} 1}=0.05 \mathrm{I}_{\max }$ and $\mathrm{I}_{\mathrm{th} 2}=0.1 \mathrm{I}_{\max }$ is presented in Fig. 10. It may be seen that for lower $\mathrm{I}_{\text {th }}$ value case (Fig. 10a), more details of the reconstructed shape may be visible than for higher $\mathrm{I}_{\text {th }}$ value (Fig. 10b). But on the other hand, if the $\mathrm{I}_{\text {th }}$ is too low, the artefacts may occur due to using the information from very dark, and possibly noised pixels, for the object local altitude slope estimation.
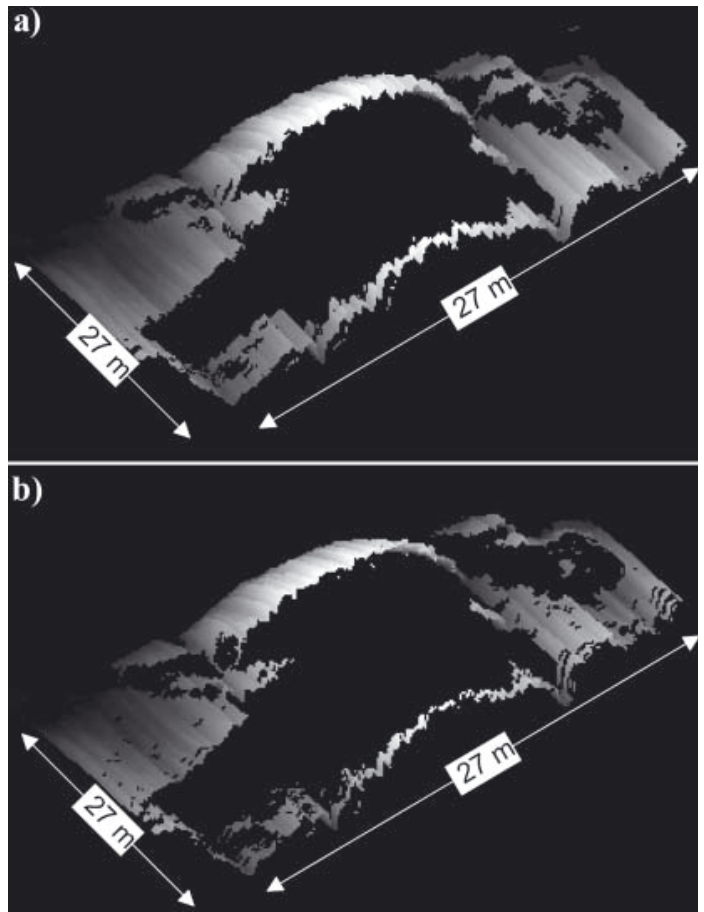

Fig. 10. $3^{D}$ wreck shape reconstruction results using two different values of shadow threshold

\section{CONCLUSIONS}

The methodology based on shape from shading approach for 3D reconstruction of seafloor and submerged objects from side scan sonar records was presented. It was verified using the data acquired by multibeam sonar system that contains both backscatter and bathymetry data and also tested on side scan sonar data. The principle advantage of the methods is their simplicity and the ability to produce the results within sequential, one-run processing. It was also shown that angular dependence of backscattering plays a significant role during image processing and should be estimated for a observed subareas.

At the current stage of the development the applicability of the presented solution might be questionable and further investigation are required. In particular, the authors expect that presented seafloor reconstruction algorithm performance can be improved by applying procedure similar to synthetic aperture sonar principle. The implementation of more advanced shape from shading algorithms could be required in more complex cases.

\section{BIBLIOGRAPHY}

1. Bikonis K., Moszynski M., Stepnowski A.: Submerged object imaging using virtual reality modeling language, Proceedings of International Congress on the Application of Recent Advances in Underwater Detection and Survey Techniques to Underwater Archeology, pp. 215-220, Bodrum, Turkey, 2004.

2. Urick J.: Principles of underwater sound for engineers, McGraw-Hill Book Company, New York, USA, 1975.

3. Bell J., Lane D., Dura E.: Automatic $3^{D}$ reconstruction of mine geometric using multiple side scan sonar images, Paper presented at GOATS 2000 Conference, SACLANT-CEN, La Spezia, Italy, 2001.

4. Cushieri J. M., M. Hebert M.: Three-dimensional map generation from side-scan sonar images, Journal of Energy Resources Technology, 112, 96-102, 1990.

5. Coiras E., Petillot Y., Lane D.: Automatic rectification of side scan sonar images, Proceedings of the $1^{\text {st }}$ International Conference on Underwater Acoustic Measurements: Technologies \& Results (CD-ROM), Heraklion, Greece, 2005.

6. Zhang R., Tsai P. S., Cryer J. E., Shah M.: Shape from shading: A survey, IEEE Transactions on Pattern Analysis and Machine Intelligence, 21, 690-705, 1999.

7. Moszynski M., Bikonis K., Lubniewski Z.: Reconstruction of $3^{D}$ shape from sidescan sonar images using Shape from Shading technique, Hydroacoustics, 16, 181-188, 2013.

8. Bikonis K., Moszynski M., Lubniewski Z., Stepnowski A.: Three-dimensional Imaging of Submerged Objects by Side-Scan Sonar Data Processing, Proceedings of the $1^{\text {st }}$ International Conference on Underwater Acoustic Measurements: Technologies \& Results (CD-ROM), Heraklion, Greece, 2005.

9. Jackson D. R., Winebrenner D. P., Ishimaru A.: Application of the composite roughness model to high-frequency bottom backscattering, J. Acoust. Am, 79(5), 1410-1422, 1986.

10.Marine Sonic Technology, Ltd. website http://www.marinesonic. us, 2012.

\section{CONTACT WITH THE AUTHORS}

Krzysztof Bikonis, Ph. D. Marek Moszynski, Assoc. Prof. Zbigniew Lubniewski, Ph. D.

Faculty of Electronics,

Telecommunications and Informatics

Gdansk University of Technology

Narutowicza 11/12

80-233 Gdansk, POLAND

e-mail: binio@eti.pg.gda.pl 